\title{
Homocysteine-induced endoplasmic reticulum stress causes dysregulation of the cholesterol and triglyceride biosynthetic pathways
}

\author{
Geoff H. Werstuck, ${ }^{1}$ Steven R. Lentz, ${ }^{2}$ Sanjana Dayal, ${ }^{2}$ Gazi S. Hossain, ${ }^{1}$ \\ Sudesh K. Sood, ${ }^{1}$ Yuan Y. Shi, ${ }^{1}$ Ji Zhou, ${ }^{1}$ Nobuyo Maeda, ${ }^{3}$ Skaidrite K. Krisans, ${ }^{4}$ \\ M. Rene Malinow, ${ }^{5}$ and Richard C. Austin ${ }^{1}$ \\ ${ }^{1}$ Department of Pathology and Molecular Medicine, McMaster University and the Hamilton Civic Hospitals Research Centre, \\ Hamilton, Ontario, Canada \\ ${ }^{2}$ Department of Internal Medicine, University of Iowa College of Medicine and Veterans Affairs Medical Center, Iowa City, \\ Iowa, USA \\ ${ }^{3}$ Department of Pathology and Program for Molecular Biology and Genetics, University of North Carolina, Chapel Hill, \\ North Carolina, USA \\ ${ }^{4}$ Department of Biology, San Diego State University, San Diego, California, USA \\ ${ }^{5}$ Oregon Regional Primate Research Center, Beaverton, Oregon, USA
}

Address correspondence to: Richard C. Austin, Hamilton Civic Hospitals Research Centre, 711 Concession Street, Hamilton, Ontario, L8V 1C3, Canada. Phone: (905) 527-2299 ext. 42628; Fax: (905) 575-2646; E-mail: raustin@thrombosis.hhscr.org.

Received for publication October 17, 2000, and accepted in revised form April 9, 2001.

\begin{abstract}
Hepatic steatosis is common in patients having severe hyperhomocysteinemia due to deficiency for cystathionine $\beta$-synthase. However, the mechanism by which homocysteine promotes the development and progression of hepatic steatosis is unknown. We report here that homocysteine-induced endoplasmic reticulum (ER) stress activates both the unfolded protein response and the sterol regulatory element-binding proteins (SREBPs) in cultured human hepatocytes as well as vascular endothelial and aortic smooth muscle cells. Activation of the SREBPs is associated with increased expression of genes responsible for cholesterol/triglyceride biosynthesis and uptake and with intracellular accumulation of cholesterol. Homocysteine-induced gene expression was inhibited by overexpression of the ER chaperone, GRP78/BiP, thus demonstrating a direct role of ER stress in the activation of cholesterol/triglyceride biosynthesis. Consistent with these in vitro findings, cholesterol and triglycerides were significantly elevated in the livers, but not plasmas, of mice having diet-induced hyperhomocysteinemia. This effect was not due to impaired hepatic export of lipids because secretion of VLDL-triglyceride was increased in hyperhomocysteinemic mice. These findings suggest a mechanism by which homocysteine-induced ER stress causes dysregulation of the endogenous sterol response pathway, leading to increased hepatic biosynthesis and uptake of cholesterol and triglycerides. Furthermore, this mechanism likely explains the development and progression of hepatic steatosis and possibly atherosclerotic lesions observed in hyperhomocysteinemia.
\end{abstract}

J. Clin. Invest. 107:1263-1273 (2001).

\section{Introduction}

Hyperhomocysteinemia is a common, independent risk factor for cardiovascular disease (1-9). Up to $40 \%$ of patients diagnosed with premature coronary artery, cerebrovascular, or peripheral vascular disease are reported to have hyperhomocysteinemia. Patients with severe hyperhomocysteinemia due to cystathionine $\beta$-synthase (CBS) deficiency also exhibit a wide range of clinical manifestations, including atherosclerosis, thrombosis, mental retardation, ectopia lentis, osteoporosis, and skeletal abnormalities. These patients also develop hepatic steatosis or "fatty liver," which is characterized by enlarged, multinucleated hepatocytes containing microvesicular lipid droplets $(10,11)$. Consistent with these findings, homozygous CBS-deficient mice having severe hyperhomocysteinemia also develop hepatic steatosis (12).
Several mechanisms have been proposed to explain the pathological changes associated with hyperhomocysteinemia. Homocysteine has been shown to cause cell injury when administered to animals $(13,14)$ or when added directly to cultured mammalian cells (15-17). Furthermore, cultured vascular endothelial cells from patients with heterozygous CBS deficiency are more sensitive to homocysteine-induced damage than are wild-type cells (17). Because homocysteine is generated intracellularly and can accumulate in cells $(18,19)$, it has been suggested that homocysteine could act intracellularly to directly modulate the activity of both large (enzymes, receptors) and small molecules (nitric oxide, glutathione) (20).

We have reported that homocysteine causes protein misfolding in the endoplasmic reticulum (ER) and activates the unfolded protein response (UPR), leading to 
increased expression of the ER stress-response genes, GRP78/BiP and GADD153 (21, 22). These observations suggest that homocysteine acts intracellularly to cause cell dysfunction by perturbing the ER. Consistent with our findings, another group has also demonstrated that homocysteine activates the UPR in cultured vascular endothelial cells, leading to increased expression of GRP78/BiP as well as two novel ER stressresponse genes, RTP and Herp $(23,24)$. However, the mechanism by which the ER stress-inducing effects of homocysteine contribute to the pathophysiology of hyperhomocysteinemia has not been established.

An association between UPR activation and lipid biosynthesis has been demonstrated in yeast $(25,26)$ and human fibroblasts (27). In concordance with these studies, we have demonstrated in cultured human vascular endothelial cells that homocysteine increases expression of the sterol regulatory element-binding protein-1 (SREBP-1) (21), an ER membrane-bound transcription factor that functions to activate genes encoding enzymes in the cholesterol/triglyceride biosynthesis and uptake pathways $(28,29)$. However, it is unknown whether homocysteine alters the expression of SREBP-dependent genes that are involved in cholesterol/triglyceride biosynthesis or whether this effect of homocysteine influences lipid metabolism in vivo.

In this study, we used cultured human cells and murine models of hyperhomocysteinemia to test the hypothesis that homocysteine-induced ER stress alters cholesterol/triglyceride metabolism. Our results show that activation of the UPR by homocysteine induces SREBP-dependent genes that are essential for biosynthesis and uptake of cholesterol and triglycerides and leads to the accumulation of cholesterol in cultured cells. Furthermore, a significant increase in the levels of cholesterol and triglycerides were observed in the livers, but not plasmas, of hyperhomocysteinemic mice. These results indicate that homocysteine-induced ER stress leads to the transcriptional activation of genes responsible for lipogenesis that likely contribute to hepatic steatosis in hyperhomocysteinemia.

\section{Methods}

Cell culture and treatment conditions. Primary human umbilical vein endothelial cells (HUVECs) were isolated by collagenase treatment of human umbilical veins (30) and cultured in M199 medium (Life Technologies Inc., Burlington, Ontario, Canada) containing 20 $\mu \mathrm{g} / \mathrm{ml}$ endothelial cell growth factor, $90 \mu \mathrm{g} / \mathrm{ml}$ porcine intestinal heparin, $100 \mu \mathrm{g} / \mathrm{ml}$ penicillin, $100 \mu \mathrm{g} / \mathrm{ml}$ streptomycin, and 20\% FBS (HyClone Laboratories, Logan, Utah, USA). Cells from passages two to four were used in these studies. Human aortic smooth muscle cells (HASMCs) were purchased from Cascade Biologics (Portland, Oregon, USA) and cultured in M231 media (Cascade Biologics) containing smooth muscle cell growth supplement (Cascade Biologics). The human hepatocarcinoma cell line, HepG2, was obtained from the American Type Culture Collection
(ATCC; Rockville, Maryland, USA) and cultured in $\alpha$ DMEM (Life Technologies Inc.) containing 10\% FBS. The human transitional bladder carcinoma cell line, T24/83, was obtained from ATCC and cultured in M199 medium containing 10\% FBS. All cells were maintained in a humidified incubator at $37^{\circ} \mathrm{C}$ with $5 \%$ $\mathrm{CO}_{2}$. DL-Homocysteine, L-methionine, DL-cysteine, glycine, DTT, tunicamycin, A23187, and $\beta$-mercaptoethanol were purchased from Sigma Chemical Co. (St. Louis, Missouri, USA). These compounds were prepared fresh in culture medium, sterilized by filtration, and added to the cell cultures.

Determination of intracellular levels of homocysteine. HepG 2 cells exposed to 1 or $5 \mathrm{mM}$ homocysteine for 0 to 24 hours were washed three times in $\alpha$-DMEM and three times in PBS. Cells were lysed in water by three freeze/thaw cycles and cellular debris removed by centrifugation. Total homocysteine, defined as the total concentration of homocysteine after quantitative reductive cleavage of all disulfide bonds (31), was determined in cellular lysates using the IMx System (Abbott Laboratories, Mississauga, Ontario, Canada) and normalized to total protein concentration.

Animals and diets. Wild-type C57BL/6 mice $\left(\mathrm{CBS}^{+/+}\right)$ were obtained from Charles River (Montreal, Québec, Canada) or The Jackson Laboratory (Bar Harbor, Maine, USA). Heterozygous $\mathrm{CBS}$-deficient mice $\left(\mathrm{CBS}^{+/-}\right)$ (12) were crossbred to $C B S^{+/+}$mice. Genotyping for the targeted allele was performed by PCR (12). At the time of weaning, offspring were fed one of four diets: (a) a control diet that contained $4.1 \mathrm{~g}$ L-methionine $/ \mathrm{kg}$ and $7.5 \mathrm{mg}$ folate/kg (LM-485; Harlan Teklad Laboratory, Madison, Wisconsin, USA); (b) a high-methionine diet that was identical to the control diet except that the drinking water was supplemented with $0.5 \%$ L-methionine; (c) a high-methionine/low-folate diet that contained $1.5 \mathrm{mg}$ folate $/ \mathrm{kg}$ and drinking water that was supplemented with $0.5 \%$ L-methionine; or (d) a very high-methionine $(24.6 \mathrm{~g} / \mathrm{kg})$, low-folate $(1.5 \mathrm{~g} / \mathrm{kg})$ diet (TD98272; Harlan Teklad Laboratory) (32). Succinylsulfathiazole $(1.0 \mathrm{mg} / \mathrm{kg})$ was added to the low-folate diets. After 2 to 20 weeks on an experimental diet, mice were sacrificed with an intraperitoneal injection of 75 mg sodium pentobarbital, plasma was collected in EDTA (final concentration 5-10 mM) for measurement of total homocysteine, and their tissues removed and snap-frozen in liquid $\mathrm{N}_{2}$ before storage at $-70^{\circ} \mathrm{C}$. Plasma total homocysteine was measured by HPLC and electrochemical detection as described previously (33). The experimental protocols were approved by the University of Iowa and Veterans Affairs Animal Care and Use Committees or the McMaster University Animal Research Ethics Board.

Hepatic VLDL-triglyceride secretion rate. $\mathrm{CBS}^{+/+}$mice fed control or hyperhomocysteinemic diets for 2 weeks were fasted overnight and hepatic VLDL-triglyceride secretion rates quantified by the intravenous administration of Triton WR1339 $(500 \mathrm{mg} / \mathrm{kg}$ body weight; Sigma Chemical Co.) as described previously (34). 
Blood samples $(50 \mu \mathrm{l})$ were taken at 0,2 , and 4 hours after Triton WR1339 injection, and plasma cholesterol and triglycerides were measured enzymatically as described below. Hepatic cholesterol and triglyceride secretion rates were calculated from the slope of the curve and expressed as micromole per hour per kilogram of body weight.

Histological analysis. Liver tissue from mice fed control or hyperhomocysteinemic diets for 16 to 20 weeks was fixed in $4 \%$ formalin, embedded in paraffin, and tissue sections were stained with hematoxylin and eosin, as described previously (35).

Preparation of total RNA. Total RNA was isolated from cultured cells or tissues using the RNeasy total RNA kit (QIAGEN Inc., Mississauga, Ontario, Canada) and resuspended in diethyl pyrocarbonate-treated water. Quantification and purity of the RNA was assessed by $\mathrm{A}_{260} / \mathrm{A}_{280}$ absorption, and RNA samples with ratios above 1.6 were stored at $-70^{\circ} \mathrm{C}$ for further analysis.

Northern blot analysis. Total RNA $(10 \mu \mathrm{g} / \mathrm{lane})$ was size fractionated on $2.2 \mathrm{M}$ formaldehyde $/ 1.2 \%$ agarose gels, transferred to Zeta-Probe GT nylon membranes (BioRad Laboratories Inc., Mississauga, Ontario, Canada), and hybridized using radiolabeled cDNA probes as described previously $(21,22)$. Signal intensities were quantified by densitometric scanning of the autoradiograms using the ImageMaster VDS and Analysis Software (Amersham Pharmacia Biotech, Baie d'Urfé, Québec, Canada). To correct for differences in gel loading, integrated optical densities were normalized to human GAPDH. The human isopentenyl diphosphate:dimethylallyl diphosphate (IPP) isomerase cDNA encodes an 837-bp DNA fragment from the $3^{\prime}$-untranslated region of the IPP isomerase gene. The cDNA probes encoding human 3-hydroxy-3-methylglutaryl coenzyme A (HMG-CoA) reductase and farnesyl diphosphate (FPP) synthase were kindly provided by Skaidrite Krisans (San Diego State University, San Diego, California, USA). Human SREBP-1 cDNA (number AA568572) was purchased from Genome Systems (St. Louis, Missouri, USA), and LDL receptor cDNA was purchased from ATCC. The cDNA probes encoding GRP78/BiP or GADD153 have been described previously $(21,22)$.

Construction of mammalian expression plasmid encoding buman GRP78/BiP. The cDNA encoding the open reading frame of human GRP78/BiP (approximately 1.95 $\mathrm{kb}$ ) was amplified by RT-PCR using total RNA from primary HUVECs. Primers used for RT-PCR were synthesized at the Institute for Molecular Biology, McMaster University. GRP78/BiP cDNA was generated using SuperScript RNase $\mathrm{H}^{-}$reverse transcriptase (Life Technologies Inc.) and a primer complimentary to a sequence in the $3^{\prime}$-untranslated region of the human GRP78/BiP mRNA transcript (AB10230: 5'TAT TAC AGC ACT AGC AGA TCA GTG-3'). For PCR amplification, the forward primer AB10231 (5'-CTT AAG CTT GCC ACC ATG AAG CTC TCC CTG GTG GCC GCG-3') contained a Kozak consensus sequence (boldface) prior to the initiating ATG and a terminal HindIII restriction site (underlined). The reverse primer AB10232 (5'-AGG CCT CGAG CT ACA ACT CAT CTT TTT CTG CTG T- $3^{\prime}$ ) contained a terminal $X h o I$ restriction site (underlined) adjacent to the authentic termination codon of the GRP78/BiP cDNA. After PCR was performed, the amplified GRP78/BiP cDNA was gel-purified using the QIAEX gel extraction kit (QIAGEN Inc.) and ligated into Tended pBluescript KS (Stratagene, La Jolla, California, USA). The ligation mixture was then used to transform competent DH5 $\alpha$ cells (Life Technologies Inc.). Plasmid DNA was isolated from transformed cells using the QIAEX miniprep kit (QIAGEN Inc.), digested with HindIII and XhoI, and the GRP78/BiP cDNA purified from agarose, as described above. The GRP78/BiP cDNA was ligated into the HindIII/XhoI site of the mammalian expression vector pcDNA3.1(+) (Invitrogen Corp., Carlsbad, California, USA) to produce the recombinant plasmid, pcDNA3.1(+)GRP78/BiP. Authenticity of the human GRP78/BiP cDNA sequence was confirmed by fluorescence-based double-stranded DNA sequencing.

Establishment of stable T24/83 cell lines overexpressing GRP78/BiP. T24/83 cells grown to $30 \%$ confluence were transfected with $5 \mu \mathrm{g}$ of the pcDNA3.1(+)GRP78/BiP expression plasmid using $30 \mu \mathrm{l}$ of SuperFect transfection reagent (QIAGEN Inc.), as described by the manufacturer. As a vector control, T24/83 cells were transfected with pcDNA3.1(+) under the same conditions. Stable transfectants were selected in complete medium containing $1.2 \mathrm{mg} / \mathrm{ml} \mathrm{G} 418$ (Life Technologies Inc.) for 2 weeks. G418-resistant clones were subsequently isolated and cultured in complete medium containing $1.0 \mathrm{mg} / \mathrm{ml} \mathrm{G} 418$. Overexpression of GRP78/BiP was assessed using immunoblotting and indirect immunofluorescence, as described below.

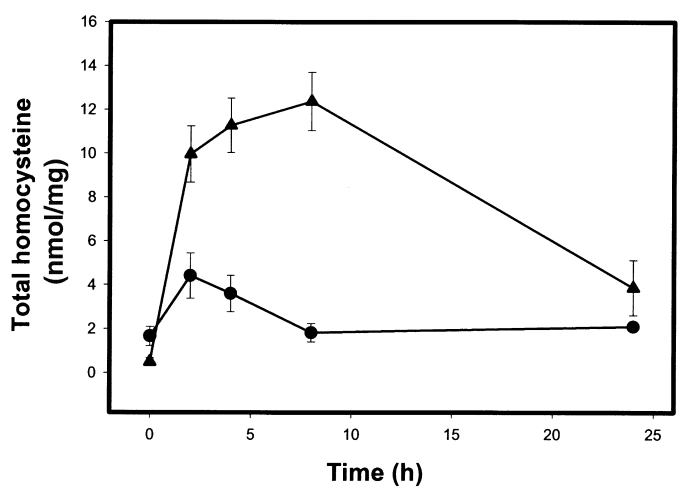

Figure 1

Intracellular homocysteine levels in HepG2 cells. HepG2 cells were cultured in the absence or presence of $1 \mathrm{mM}$ (circles) or $5 \mathrm{mM}$ (triangles) homocysteine for the indicated time periods, washed, and lysed by three freeze/thaw cycles. Total intracellular homocysteine was determined and normalized to total protein. Data are presented as the mean \pm SD of three separate experiments. 
a
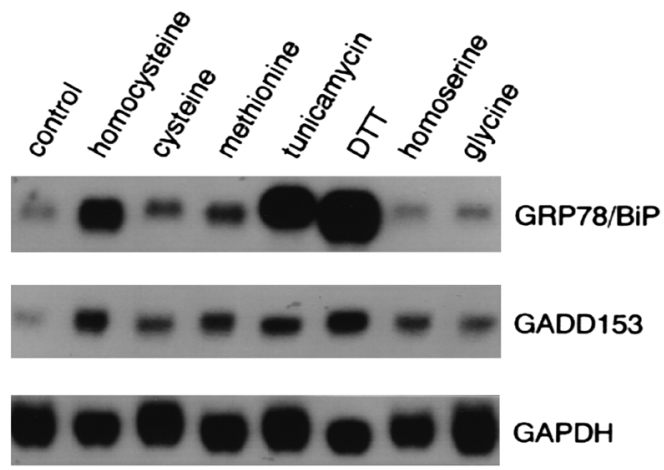

b

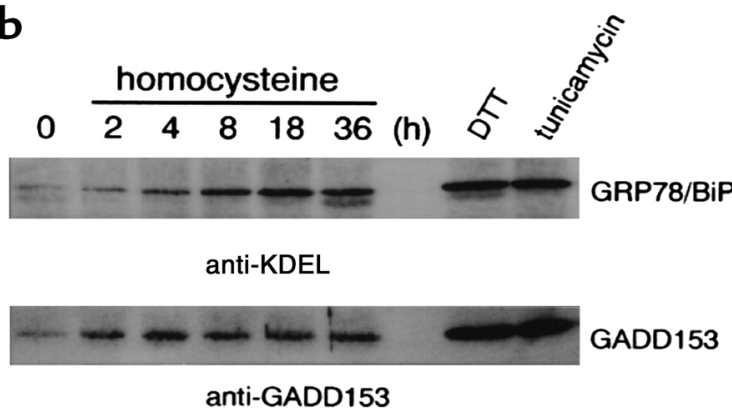

Immunoblot analysis. The anti-KDEL mAb (SPA827), which recognizes both GRP78/BiP and GRP94, was purchased from StressGen Biotechnologies Corp. (Victoria, British Columbia, Canada). The anti-GADD153 polyclonal Ab (sc-575) was purchased from Santa Cruz Biotechnology Inc. (Santa Cruz, California, USA). The mAb's reactive against human SREBP-1 or -2 (clones IgG-2A4 and IgG-1C6, respectively) were purchased from PharMingen (Mississauga, Ontario, Canada). Total protein lysates from mouse tissues or cultured cells were solubilized in SDS-PAGE sample buffer and separated on SDS-polyacrylamide gels under reducing conditions, as described previously $(21,22)$. After incubation with the appropriate primary and horseradish peroxidase-conjugated (HRP-conjugated) secondary Ab's (Life Technologies Inc.), the membranes were developed using the SuperSignal chemiluminescent substrate (Pierce Chemical Co., Rockford, Illinois, USA).

\section{Figure 3}

Homocysteine induces the expression of SREBP-1 in HepG2 cells. (a) Immunoblot analysis of SREBP-1 protein in HepG2 cells cultured in the absence or presence of 5 $\mathrm{mM}$ homocysteine for the indicated time periods. Total protein lysates ( $40 \mu \mathrm{g} / \mathrm{lane})$ were separated on $10 \%$ SDS-polyacrylamide gels under reducing conditions, transferred to nitrocellulose membranes, and immunostained with an $\mathrm{mAb}$ that recognizes both the precursor (P) and mature (M) forms of SREBP-1. (b) Northern blot analysis of the steady-state mRNA levels of SREBP-1 in HepG2 cells cultured in the presence of $5 \mathrm{mM}$ homocysteine for the indicated time periods. Total RNA (10 $\mu \mathrm{g} /$ lane) was size fractionated by agarose-gel electrophoresis, transferred to nylon membranes, and subjected to blot hybridization using a radiolabeled cDNA probe encoding human SREBP-1. Control for equivalent RNA loading was assessed using a radiolabeled GAPDH cDNA probe.
Immunohistochemistry and image analysis. Immunohistochemistry using polyclonal Ab's against GRP78/BiP (sc-1050; Santa Cruz Biotechnology Inc.) was performed as described previously $(21,22)$. Images were captured with Northern Eclipse image analysis/archival software (Empix Imaging Inc., Mississauga, Ontario, Canada).

Uptake of BODIPY FL LDL. Cells treated in the absence or presence of homocysteine were washed with PBS and incubated in media containing 10 $\mu \mathrm{g} / \mathrm{ml}$ BODIPY FL LDL (Molecular Probes, Eugene, Oregon, USA). After incubation at $37^{\circ} \mathrm{C}$ for 2 hours, cells were washed with PBS, fixed in $3 \%$ formaldehyde in PBS, and the uptake of LDL was detected by fluoce microscopy.

tot cholesterol and triglyceride levels. Cultured cells or tissues were homogenized in lysis buffer containing $0.1 \%$ Triton X-100. Lysates were saponified, and lipids were extracted with hexane/isopropanol (3:2) (36). Colorimetric cholesterol and triglyceride assays were carried out using the Sigma Diagnostics Cholesterol and
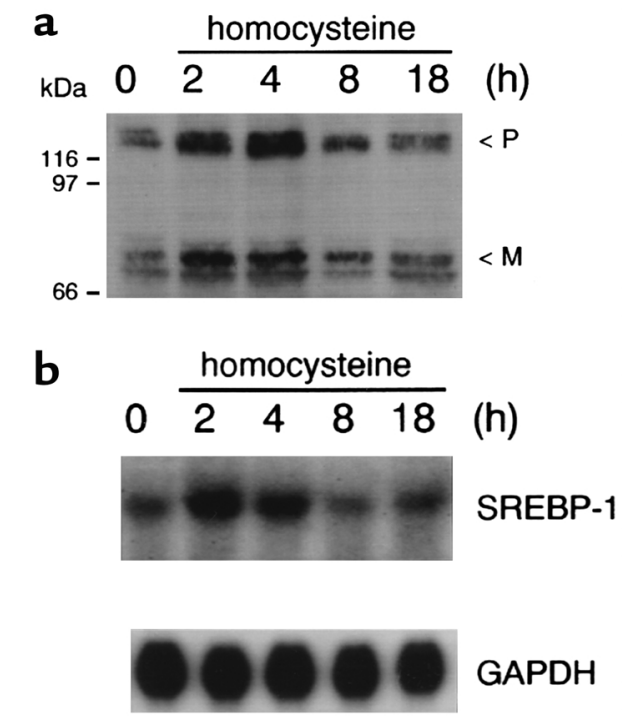

GAPDH 


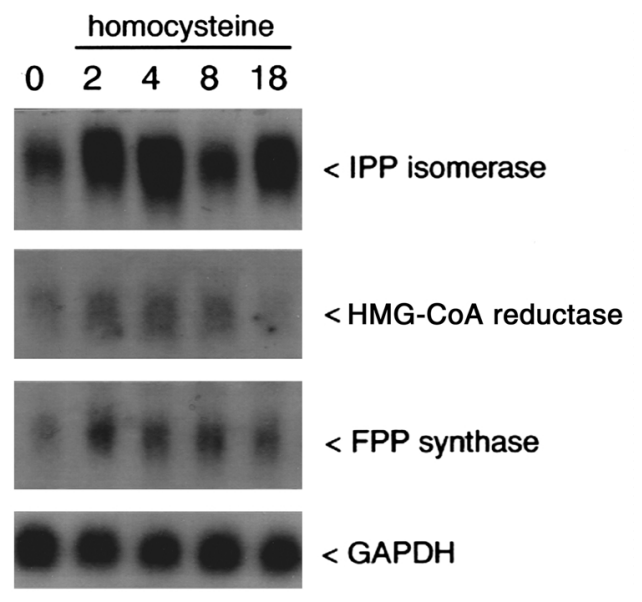

\section{Figure 4}

Homocysteine induces the steady-state mRNA levels of genes involved in cholesterol biosynthesis in HepG2 cells. Northern blot analysis of the steady-state mRNA levels of IPP isomerase, HMG-CoA reductase, and FPP synthase in HepG2 cells cultured in the absence or presence of $5 \mathrm{mM}$ homocysteine for the indicated time periods. Total RNA (10 $\mu \mathrm{g} /$ lane) was size fractionated by agarose-gel electrophoresis, transferred to nylon membranes, and subjected to blot hybridization using the appropriate radiolabeled cDNA probe. Control for equivalent RNA loading was assessed using a radiolabeled GAPDH cDNA probe.

Triglyceride Reagents (Sigma Chemical Co.). Total plasma cholesterol and triglycerides were measured using the same assays, but without the lipid-extraction step. Statistical analysis. Results are presented as the mean plus or minus SD. Differences in treatment groups were analyzed using two-way ANOVA. On finding significance with ANOVA, unpaired Student's $t$ test was performed. For all analyses, $P$ values less than 0.05 were considered statistically significant.

\section{Results}

Intracellular levels of homocysteine in HepG2 cells. We have demonstrated previously that intracellular levels of homocysteine are transiently increased in HUVECs exposed to exogenous homocysteine (22). To increase intracellular homocysteine levels in HepG2 cells, varying concentrations of DL-homocysteine up to $5 \mathrm{mM}$ were added to the cell cultures. Figure 1 shows that to attain a twofold to sixfold transient increase in intracellular homocysteine, HepG2 cells require an extracellular homocysteine concentration of 1 to $5 \mathrm{mM}$. These extracellular homocysteine concentrations did not affect overall cell number or viability as determined by trypan blue and ${ }^{51} \mathrm{Cr}$-release assays (data not shown).

Homocysteine causes ER stress in HepG2 cells. We have shown in HUVECs that homocysteine causes ER stress, leading to activation of the UPR and increased expression of GRP78/BiP and GADD153 $(21,22)$. As shown in Figure 2a, homocysteine increased the steady-state mRNA levels of GRP78/BiP (6.7-fold) and GADD153 (3.4-fold) in HepG2 cells $(P<0.05)$. This effect was selective for homocysteine because other structurally related amino acids such as methionine, cysteine, homoserine, and glycine did not significantly induce the expression of these genes. In addition to homocysteine, other agents known to cause ER stress, including DTT and tunicamycin, increased the steady-state mRNA levels of GRP78/BiP and GADD153 in HepG2 cells $(P<0.05)$. Consistent with these findings, GRP78/BiP and GADD153 protein levels were also elevated in HepG2 cells after treatment with homocysteine, tunicamycin, or DTT (Figure 2b).

Induction of ER stress by homocysteine increases the expression of SREBPs and genes encoding enzymes in the cholesterol/triglyceride biosynthetic pathway. Immunoblot analysis showed that HepG2 cells had increased levels of both mature $(68 \mathrm{kDa})$ and precursor $(125 \mathrm{kDa})$ forms of SREBP-1 after treatment with homocysteine for 2 and 4 hours (Figure 3a). Mature and precursor forms of SREBP-2 were also increased in HepG2 cells by homocysteine (data not shown). Because activated SREBPs induce (autoregulate) expression of precursor SREBPs, in addition to regulating genes involved in cholesterol/triglyceride biosynthesis and uptake $(28,29,37$, 38), Northern blots were used to examine the effect of homocysteine on the steady-state mRNA levels of SREBP-1 and several genes encoding key enzymatic components of the cholesterol/triglyceride biosynthetic pathway. Steady-state mRNA levels of SREBP-1 were increased and peaked between 2 and 4 hours after treatment with homocysteine (Figure 3b). Furthermore, steady-state mRNA levels of genes encoding enzymes of the cholesterol biosynthetic pathway, including HMGCoA reductase, IPP isomerase, and FPP synthase, were increased and peaked between 2 and 4 hours in HepG 2 cells after treatment with homocysteine (Figure 4). Steady-state mRNA levels for the LDL receptor, as well
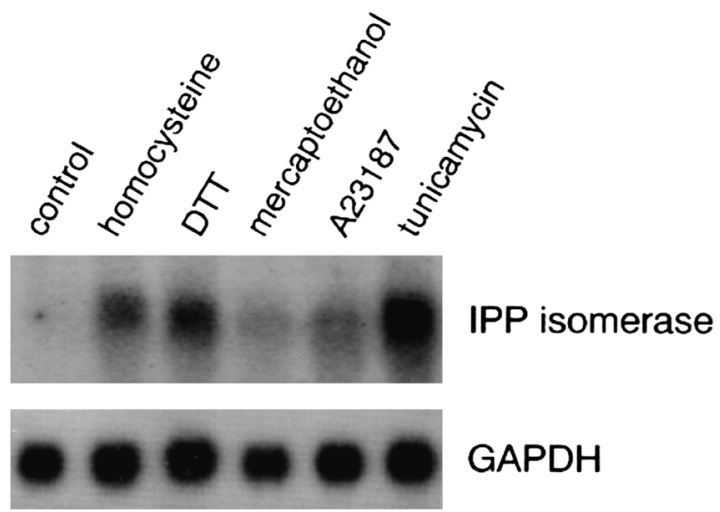

\section{Figure 5}

Effect of ER stress agents on steady-state mRNA levels of IPP isomerase in HepG2 cells. Equivalent amounts of total RNA (10 $\mu \mathrm{g} / \mathrm{lane})$ isolated from HepG2 cells cultured for 4 hours in the absence (control) or presence of $5 \mathrm{mM}$ homocysteine, $2.5 \mathrm{mM}$ DTT, $5 \mathrm{mM} \beta$-mercaptoethanol, $10 \mu \mathrm{M}$ of the $\mathrm{Ca}^{2+}$ ionophore $\mathrm{A} 23187$, or $10 \mu \mathrm{g} / \mathrm{ml}$ tunicamycin were examined by Northern blot analysis using a radiolabeled IPP isomerase CDNA probe. Control for equivalent RNA loading was assessed using a radiolabeled GAPDH cDNA probe. 


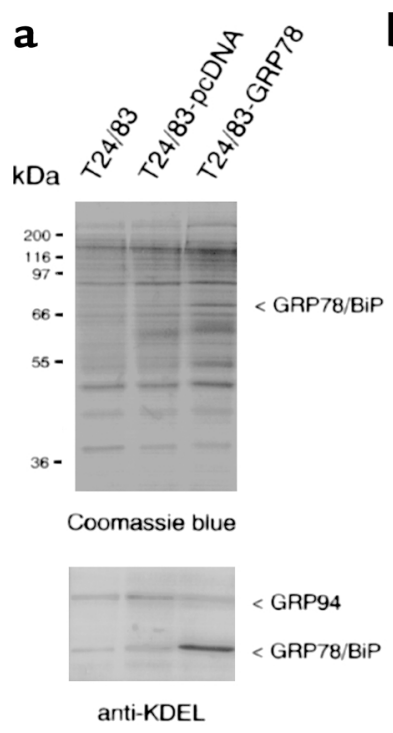

b

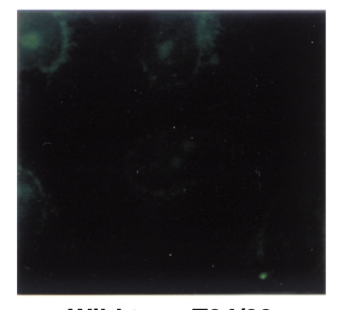

Wild-type T24/83

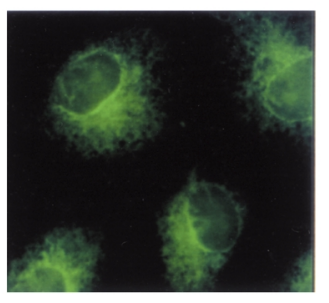

T24/83-GRP78
C

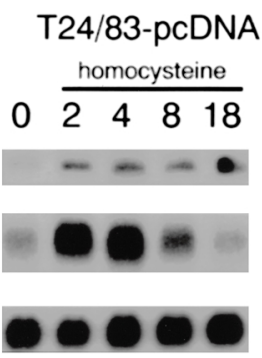

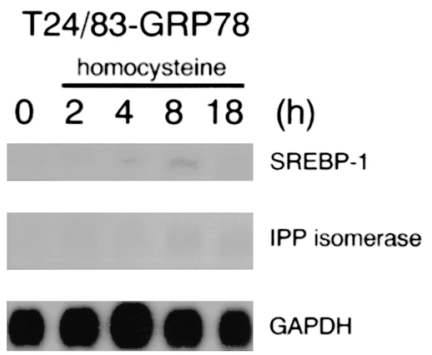

Figure 6

Overexpression of GRP78/BiP prevents the increase in steady-state mRNA levels of SREBP-1 and IPP isomerase by homocysteine. (a) Immunoblot analysis of GRP78/BiP and GRP94 in wild-type (T24/83), vector-transfected (T24/83-pcDNA), or GRP78/BiP-overexpressing (T24/83-GRP78) cells. Total protein lysates (40 $\mu \mathrm{g} /$ lane) were separated on $10 \%$ SDS-polyacrylamide gels under reducing conditions and either stained with Coomassie blue (upper panel) or immunostained with an anti-KDEL mAb. (b) Immunolocalization of GRP78/BiP in the ER of wild-type or GRP78/BiP-overexpressing T24/83 cells. Cells grown on glass coverslips were fixed, permeabilized, and immunostained with an anti-GRP78/BiP polyclonal Ab. $\times 800$. (c) Northern blot analysis of the steady-state mRNA levels of SREBP-1 and IPP isomerase in vector-transfected (T24/83-pcDNA) or GRP78/BiP-overexpressing (T24/83-GRP78) cells cultured in the absence or presence of $1 \mathrm{mM}$ homocysteine for the indicated time periods. Total RNA ( $10 \mu \mathrm{g} /$ lane) was size fractionated by agarosegel electrophoresis, transferred to nylon membranes, and subjected to blot hybridization using radiolabeled cDNA probes encoding human SREBP-1 or IPP isomerase. Control for equivalent RNA loading was assessed using a radiolabeled GAPDH cDNA probe.

as acetyl CoA carboxylase and fatty acid synthase, genes encoding enzymes involved in fatty acid synthesis, were also increased in homocysteine-treated HepG2 cells (data not shown). Similar patterns of gene induction were also observed in HASMCs and HUVECs exposed to homocysteine (data not shown). Addition of cycloheximide to cells exposed to homocysteine did not inhibit the induction of these genes (data not shown), a finding consistent with a mechanism involving the activation of existing precursor SREBPs $(28,29)$.
Induction of IPP isomerase gene expression by ER stress. HepG2 cells were treated with ER stress-inducing agents, including tunicamycin, DTT, $\beta$-mercaptoethanol, and the calcium ionophore, A23187, and Northern blot analysis was used to examine changes in IPP isomerase gene expression. To varying degrees, all ER stress agents tested induced the expression of IPP isomerase, compared with untreated cells (Figure 5). Similar results were also observed for HASMCs and HUVECs treated with ER stress agents (data not shown).

Overexpression of GRP78/BiP prevents the induction of SREBP-1 and IPP isomerase genes by homocysteine. Overexpression of GRP78/BiP has been reported to protect cells from ER stress $(39,40)$. Stably transfected cells overexpressing GRP78/BiP were generated to assess whether blocking homocysteine-induced ER stress attenuated the expression of SREBP-1 and IPP isomerase. As shown in Figure 6a, the GRP78/BiP-overexpressing cells exhibited a threefold increase in GRP78/BiP protein levels, compared with wild-type or vector-transfected cells. Furthermore, GRP78/BiP was localized to the ER at a much greater intensity, compared with wild-type cells (Figure 6b). When treated with homocysteine, the steady-state mRNA levels of SREBP-1 and IPP isomerase were dramatically decreased in the GRP78/BiP-overexpressing cells, compared with vector-transfected (Figure 6c) or wild-type cells (data not shown). These findings indicate that overexpression of GRP78/BiP inhibits the induction of SREBP-1 and IPP isomerase gene expression by homocysteine and suggests that ER stress plays an important role in the activation of genes responsible for cholesterol/triglyceride biosynthesis.

Effect of homocysteine on cellular levels of cholesterol. To determine whether the homocysteine-mediated induction of genes responsible for cholesterol biosynthesis is associated with a corresponding increase in intracellular cholesterol, HUVECs, HASMCs, and HepG2 cells were cultured in the absence or presence of either homocysteine or cysteine for 24 and 48 hours, and total cholesterol was determined. Incubation with 1 or 5 $\mathrm{mM}$ homocysteine increased cellular cholesterol in HepG2 and HASMCs (Figure 7). In contrast, cholesterol levels were unchanged in HUVECs, despite increased expression of SREBP-1 and genes encoding enzymes in the cholesterol biosynthetic pathway. Incubation with cysteine did not influence levels of cholesterol in any of the cell types (data not shown).

Effect of homocysteine on LDL uptake. In addition to their effects on cholesterol/triglyceride biosynthesis, the SREBPs also regulate LDL-receptor expression and activity $(28,29)$. To explore the effect of homocysteine on cholesterol uptake via the LDL receptor, we measured the ability of cultured cells treated with homocysteine to bind and internalize fluorescently labeled LDL (Figure 8). Our results indicate that after incubation with homocysteine, HASMCs maintained their ability to endocytose LDL while HepG2 cells showed enhanced LDL uptake. These results suggest that 


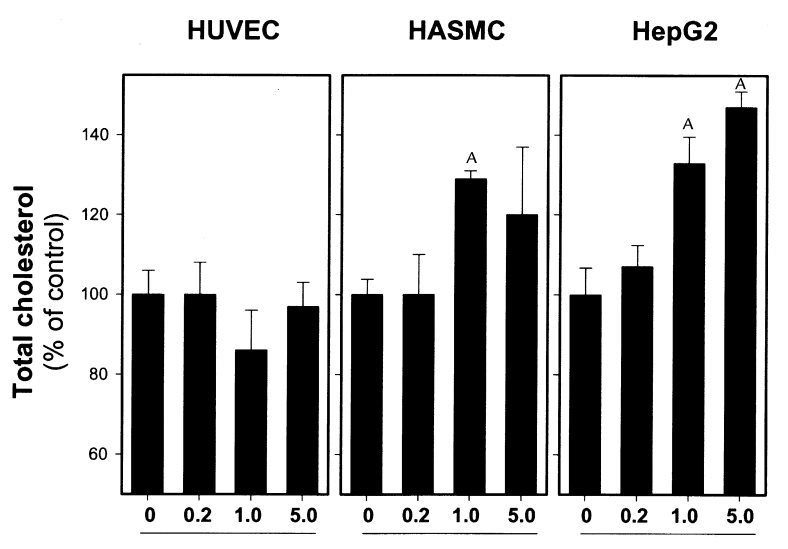

Homocysteine (mM)

upregulation of the cholesterol/triglyceride biosynthetic pathway in HASMCs and HepG2 cells does not result from impaired LDL uptake. In contrast, HUVECs treated with homocysteine showed a significant decrease in LDL uptake, which may explain why these cells do not accumulate cholesterol and triglycerides when incubated with homocysteine.

Cholesterol levels in mice with diet-induced hyperhomocysteinemia. To determine the effect of hyperhomocysteinemia on lipid metabolism in vivo, we measured cholesterol and triglycerides in the livers and plasmas of $\mathrm{CBS}^{+/+}$and $\mathrm{CBS}^{+/-}$mice fed control or hyperhomocysteinemic diets for 10 to 20 weeks. Compared with age-matched mice fed control diet, $\mathrm{CBS}^{+/+}$mice fed the high-methionine/low-folate diet had markedly elevated levels of hepatic cholesterol and triglycerides (Table 1). Hepatic cholesterol also was elevated modestly in $\mathrm{CBS}^{+/+}$mice fed the high-methionine diet. Compared with $\mathrm{CBS}^{+/+}$mice fed the same diets, $\mathrm{CBS}^{+/-}$mice exhibited similar hepatic triglyceride levels and slightly increased hepatic cholesterol levels. Plasma cholesterol levels tended to be elevated in $\mathrm{CBS}^{+/+}$and $\mathrm{CBS}^{+/-}$mice fed the high-methionine/low-folate diet compared with

\section{Figure 7}

Homocysteine increases intracellular total cholesterol in cultured human cells. HUVECs, HASMCs, and HepG2 cells were incubated for 48 hours in media containing $0,0.2,1$, or $5 \mathrm{mM}$ homocysteine. Cells were washed in PBS, harvested in $0.2 \mathrm{M} \mathrm{NaOH}$, and lipids extracted as described in Methods. Total cholesterol was normalized for protein content, and values were expressed as a percentage versus cells treated in the absence of homocysteine. Data are presented as the mean \pm SD from three separate experiments. ${ }^{A} P<0.05$ between control and homocysteine-treated cells.

mice fed control diet, but these differences did not reach statistical significance. No differences in plasma triglycerides were detected between groups. Histological analysis of liver sections from $\mathrm{CBS}^{+/+}$and $\mathrm{CBS}^{+/-}$ mice fed high-methionine/low-folate diet revealed that their hepatocytes were engorged with lipid vesicles (Figure 9). All mice with diet-induced hyperhomocysteinemia appeared normal and their body weights were similar to those of mice fed control diets.

VLDL-triglyceride secretion rate in mice fed control or byperhomocysteinemic diet. To assess whether the accumulation of hepatic cholesterol and triglycerides were due to impaired lipid export, VLDL-triglyceride secretion rate was determined by injecting $C B S^{+/+}$mice that had been fasted overnight with Triton WR1339. Because Triton WR1339 blocks conversion of VLDL to LDL, the resulting increase in plasma triglyceride and cholesterol concentrations reflect hepatic secretion of VLDL in the absence of LDL uptake (34). Figure 10 shows that the hepatic triglyceride secretion rate was significantly higher in $\mathrm{CBS}^{+++}$mice fed hyperhomocysteinemic diets, compared with $\mathrm{CBS}^{+++}$mice fed control diet. Furthermore, the triglyceride secretion rate was highest in mice fed the very high-methionine/low-folate diet, which produced the highest levels of plasma total homocysteine ( $334 \pm 100$ $\mu \mathrm{M})$. Cholesterol secretion rate was also significantly elevated in these mice. These results suggest that accumulation of hepatic cholesterol and triglycerides in hyperhomocysteinemia is related to increased biosynthesis and uptake rather than decreased hepatic export of VLDL.

Table 1

Comparison of cholesterol and triglyceride levels in the livers and plasmas of $\mathrm{CBS}^{+/+}$and $\mathrm{CBS}^{+/-}$mice fed control or hyperhomocysteinemic diets

\begin{tabular}{|c|c|c|c|c|c|c|}
\hline \multirow[b]{2}{*}{ Genotype } & \multirow[b]{2}{*}{$\operatorname{Diet}^{\mathrm{A}}$} & \multicolumn{3}{|c|}{ Plasma } & \multicolumn{2}{|c|}{ Liver } \\
\hline & & $\begin{array}{c}\text { homocysteine }^{B} \\
(\mu \mathrm{M})\end{array}$ & $\begin{array}{l}\text { cholesterol } \\
(\mathrm{mM})\end{array}$ & $\begin{array}{l}\text { triglycerides } \\
(\mathrm{mM})\end{array}$ & $\begin{array}{c}\text { cholesterol }^{\mathrm{C}} \\
(\mathrm{mg} / \mathrm{mg} \text { protein })\end{array}$ & $\begin{array}{c}\text { triglycerides } \mathrm{C} \\
(\mathrm{mg} / \mathrm{mg} \text { protein })\end{array}$ \\
\hline \multicolumn{7}{|l|}{$\mathrm{CBS}^{+/+}$} \\
\hline & Control & $2.5(0.9)$ & $0.91(0.49)$ & $4.8(0.8)$ & $0.018(0.006)$ & $0.10(0.02)$ \\
\hline & $\mathrm{HM}$ & $8.8(4.5)$ & $0.66(0.30)$ & $5.7(1.6)$ & $0.027(0.002)^{\mathrm{D}}$ & $0.11(0.01)$ \\
\hline & HMLF & $60(61)$ & $1.56(0.40)$ & $6.7(1.5)$ & $0.16(0.04)^{\mathrm{D}}$ & $0.69(0.31)^{\mathrm{D}}$ \\
\hline \multicolumn{7}{|l|}{$\mathrm{CBS}^{+/-}$} \\
\hline & Control & $6.2(3.8)$ & $0.93(0.45)$ & $6.0(1.0)$ & $0.026(0.003)^{\mathrm{E}}$ & $0.11(0.03)$ \\
\hline & $\mathrm{HM}$ & $27(18)$ & $0.63(0.27)$ & $5.5(1.6)$ & $0.025(0.001)$ & $0.12(0.02)$ \\
\hline & HMLF & $48(63)$ & $1.41(0.37)$ & $6.7(1.5)$ & $0.33(0.02)^{\mathrm{D}}$ & $0.39(0.06)^{\mathrm{D}}$ \\
\hline
\end{tabular}

AMice were fed control, $\mathrm{HM}$, or HMLF diets for 10 weeks. ${ }^{\mathrm{B}} \mathrm{All}$ data are expressed as the mean $\pm \mathrm{SD}$ ( $n=4$ to 8 mice/group). ${ }^{C}$ Liver cholesterol and triglyceride concentrations are normalized to the total protein content of the tissue. ${ }^{D} P<0.05$ between the indicated values and the corresponding controls. $E P<0.05$ between $\mathrm{CBS}^{+/-}$and $\mathrm{CBS}^{+/+}$controls. $\mathrm{HM}$, high-methionine; $\mathrm{HMLF}$, high-methionine/low-folate. 

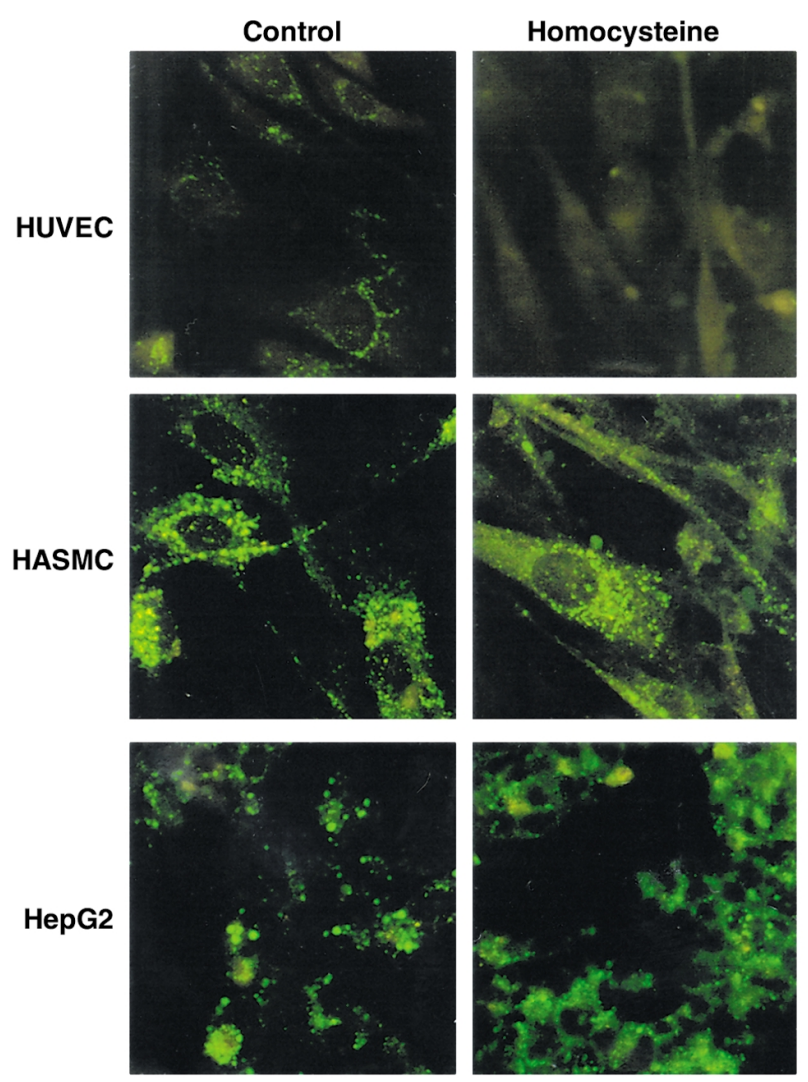

Hyperhomocysteinemic mice have increased hepatic $m R N A$ levels of GADD153, SREBP-1, and the LDL receptor. To determine whether hepatic cholesterol and triglyceride accumulation in hyperhomocysteinemic mice is associated with ER stress and activation of the UPR, we probed total RNA isolated from the livers of $\mathrm{CBS}^{+/+}$mice fed control or hyperhomocysteinemic diets for GADD153 gene expression (Figure 11), which is a direct indicator of ER stress $(41,42)$. Northern blot analysis demonstrated that steady-state mRNA levels of GADD153 were elevated 6.7-fold in mice fed high-methionine diet and 11.4-fold in mice fed high-methionine/low-folate diet for 2 weeks, compared with control mice $(P<0.05)$. This result suggests that hyperhomocysteinemia causes ER stress and UPR activation in vivo.

\footnotetext{
Figure 9

Hepatic morphology of $\mathrm{CBS}^{+/+}$and $\mathrm{CBS}^{+/-}$mice fed control diet or high-methionine/low-folate diet. $\mathrm{CBS}^{+/+}$mice fed control (a) or high-methionine/low-folate diet (c) for 20 weeks. $\mathrm{CBS}^{+/-}$mice fed control (b) or high-methionine/low-folate diet (d) for 16 weeks. The hepatocytes from mice fed the high-methionine/low-folate $\operatorname{diet}$ ( $\mathbf{c}$ and $\mathbf{d}$ ) are enlarged, multinucleated, and contain extensive microvesicular and macrovesicular lipid with no apparent fibrosis or necrosis. Hematoxylin and eosin staining. $\times 400$.
}

Figure 8

Effect of homocysteine on LDL uptake in HUVECs, HASMCs, and HepG2. Cells treated in the absence or presence of $5 \mathrm{mM}$ homocysteine for 8 hours were washed with PBS followed by incubation for an additional 2 hours at $37^{\circ} \mathrm{C}$ in media containing $10 \mu \mathrm{g} / \mathrm{ml}$ BODIPY FL LDL. After washing with PBS, cells were fixed, and LDL binding/uptake was detected by fluorescence microscopy. $\times 375$.

Northern blot analysis indicated that the steadystate mRNA levels of SREBP-1 and the LDL receptor were also significantly increased $(P<0.05)$ in the livers of $\mathrm{CBS}^{+/+}$mice with diet-induced hyperhomocysteinemia, compared with control mice (Figure 11). This result is consistent with our in vitro findings and suggests that a combination of increased endogenous cholesterol/triglyceride production and uptake lead to hepatic lipid accumulation in mice having dietinduced hyperhomocysteinemia.

\section{Discussion}

The present study provides novel evidence that homocysteine-induced ER stress enhances lipid biosynthesis and uptake through activation of the SREBPs and likely explains the development and progression of hepatic steatosis in hyperhomocysteinemia. Specifically, we have demonstrated that homocysteine-induced ER stress (a) activates the UPR leading to increased expression of GRP78/BiP and GADD153, (b) induces proteolytic cleavage of SREBP-1 and -2, (c) enhances expression of genes responsible for cholesterol/triglyceride biosynthesis and uptake, and (d) increases total cholesterol without decreasing LDL uptake in cultured hepatocytes and smooth muscle cells. Consistent with our in vitro findings, a significant increase in GADD153, SREBP-1, and LDL receptor-gene expression, as well as the accumulation of cholesterol and triglycerides, were observed in livers of mice having diet-induced hyperho- 


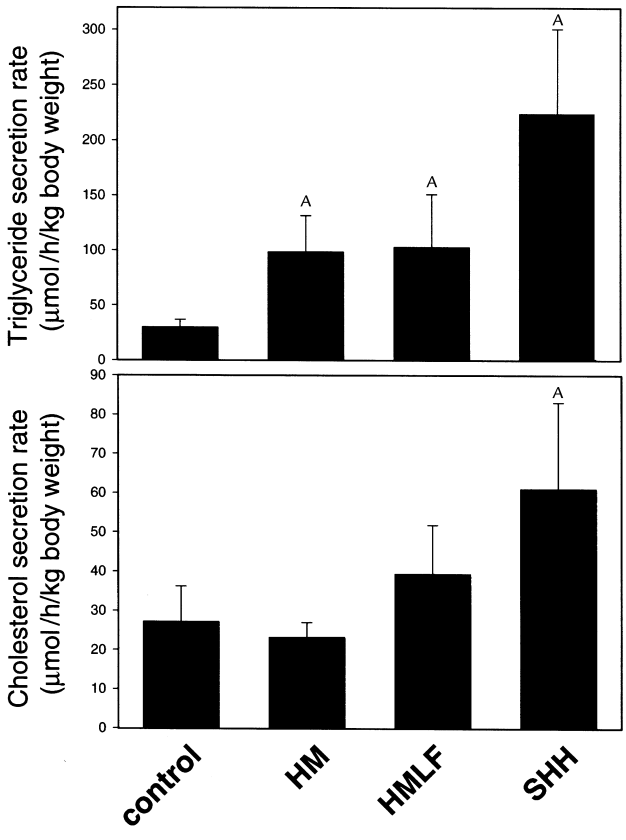

Figure 10

VLDL-triglyceride secretion rates in $\mathrm{CBS}^{+/+}$mice fed control or hyperhomocysteinemic diets. $\mathrm{CBS}^{+/+}$mice were fed a control diet, a highmethionine diet (HM), a high-methionine/low-folate diet (HMLF), or a very high-methionine/low-folate $\operatorname{diet}(\mathrm{SHH})$ for 2 weeks. Plasma VLDL-triglyceride levels were determined in mice 0,2 , and 4 hours after Triton WR1339 injection. Hepatic VLDL-triglyceride secretion rate was calculated from the slope of the curve. Data are presented as the mean $\pm \mathrm{SD}$ ( $n=4$ mice/group). Mean total plasma homocysteine concentration for mice on the $\mathrm{SHH}$ diet was $334 \pm 100 \mu \mathrm{M}$. ${ }^{A} P<0.05$ between control and mice having hyperhomocysteinemia.

mocysteinemia. The additional observation that VLDLtriglyceride secretion rates were significantly elevated in hyperhomocysteinemic mice suggests that hepatic steatosis is not due to impaired lipid export, but instead results from increased lipid biosynthesis and uptake. Although previous studies have demonstrated hepatic steatosis in homozygous $\mathrm{CBS}^{-/-}$mice having severe hyperhomocysteinemia (12), our findings provide direct evidence that hepatic steatosis can occur in $\mathrm{CBS}^{+/+}$and $\mathrm{CBS}^{+/-}$mice having diet-induced hyperhomocysteinemia and further highlight the importance of diet as a major contributor to the pathophysiological outcome of hyperhomocysteinemia.

Increased dietary methionine or deficiencies of folic acid and vitamin B6 and/or vitamin B12 can lead to hyperhomocysteinemia in humans (43-45) and animals $(32,35,46,47)$. However, the mechanisms by which elevated levels of homocysteine promote the pathological changes associated with hyperhomocysteinemia are poorly understood. Because the highly reactive thiol group of homocysteine is readily oxidized in plasma to form reactive oxygen species, it has been suggested that homocysteine exerts its cytotoxic effect through a mechanism involving oxidative damage $(15-17,48,49)$. However, the oxidative stress hypothesis fails to explain why cysteine, which is present in plasma in concentrations 25- to 30 -fold higher than those of total homocysteine and is readily oxidized, is not usually considered to be a risk factor for cardiovascular disease (20). In fact, markers of oxidative stress have not been observed in cultured human cells exposed to homocysteine (21-24) or in the livers of hyperhomocysteinemic mice (49). However, given that homocysteine decreases the expression of a wide range of antioxidant enzymes (21) and impairs the activity of glutathione peroxidase (50), homocysteine may indirectly enhance oxidative damage in certain cells or tissues by decreasing cellular antioxidant potential $(20,51)$. An additional hypothesis is that cellular dysfunction is caused by elevation of intracellular concentrations of homocysteine itself and that elevated plasma total homocysteine is a marker of increased intracellular homocysteine. To increase intracellular homocysteine levels in some cultured cells, we and others have applied exogenous homocysteine in concentrations up to $5 \mathrm{mM}(21-24)$. Though significantly higher than the pathophysiological range of plasma total homocysteine, these concentrations of homocysteine do not impair cell viability and only transiently increase intracellular levels twofold to sixfold $(21,22)$. Unlike cysteine, homocysteine causes specific intracellular effects including ER stress (21-24), decreased bioavailability of nitric oxide (50), and increased protein damage caused by homocysteinylation $(18,19)$.

We found that hepatic ER stress and UPR activation were evident when mice were fed hyperhomocysteinemic diets for 2 weeks. It is well established that agents and/or

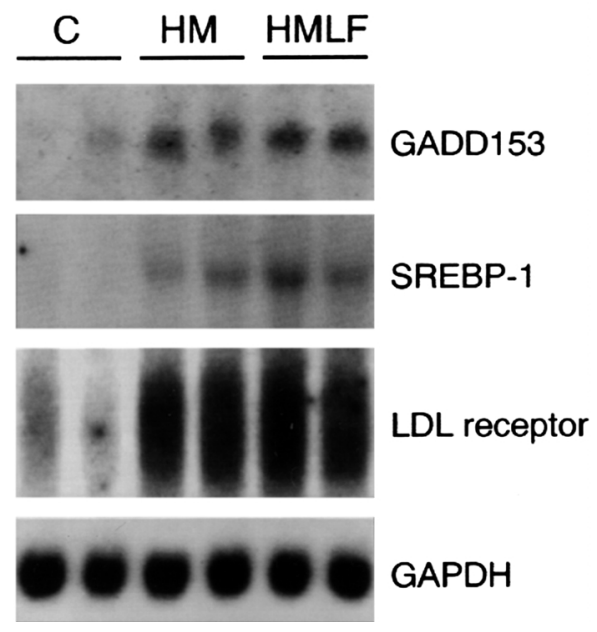

Figure 11

Increased steady-state mRNA levels of GADD153, SREBP-1, and the LDL receptor in the livers of mice having hyperhomocysteinemia. $\mathrm{CBS}^{+/+}$mice were fed a control diet (C), a high-methionine diet (HM), or a combination high-methionine/low-folate diet (HMLF). After 2 weeks, the animals were sacrificed and tissues harvested. Total RNA $(10 \mu \mathrm{g} /$ lane $)$ isolated from the livers of two randomly selected animals from each group $(n=5)$ was examined by Northern blot analysis using radiolabeled cDNA probes encoding human GADD153, SREBP-1, or LDL receptor. Control for equivalent RNA loading was assessed using a radiolabeled GAPDH cDNA probe. 
conditions that adversely affect ER function activate the UPR, resulting in increased expression of GRP78/BiP, GRP94 (52-54), GADD153 (41, 42), and activation of the ER membrane-bound transcription factor, ATF6 (55). Recent studies have indicated that the UPR regulates lipid biosynthesis in yeast $(25,26)$ and dolichol biosynthesis, which is part of the cholesterol biosynthesis pathway, in human fibroblasts (27). It is likely that the UPR coordinates the synthesis of ER chaperones as well as ER membrane components to increase the folding capacity and the space required to accommodate the accumulation of unfolded proteins. Our findings suggest that activation of the UPR by homocysteine promotes the overproduction of ER lipid components, including cholesterol and triglycerides, resulting in the accumulation of lipids within affected cells. It follows that by blocking or minimizing ER stress, it may be possible to attenuate the induction of lipid biosynthesis. In support of this concept, we observed that stable overexpression of GRP78/BiP, which protects cells from agents or conditions known to cause ER stress $(39,40)$, inhibits homocysteine-induced expression of genes involved in cholesterol biosynthesis in cultured human cells.

Despite an increase in VLDL-triglyceride secretion rate, hyperhomocysteinemic mice did not have significantly increased levels of plasma cholesterol or triglycerides. These observations suggest that hepatic steatosis in hyperhomocysteinemia does not result from impaired VLDL export, but instead is due to increased lipid biosynthesis coupled with inappropriately enhanced uptake of LDL by liver and perhaps other tissues. These findings are consistent with previous studies demonstrating increased hepatic lipid biosynthesis and VLDL secretion in transgenic mice that overexpress fully active, nuclear SREBP1a $(56,57)$. These mice also develop severe hepatic steatosis, but maintain normal plasma lipid profiles as a result of SREBP-induced LDL receptor activity. The localized accumulation of lipid in tissues, such as liver, that are sensitive to ER stress provides a plausible explanation as to the prevalence of hepatic steatosis in hyperhomocysteinemic patients having relatively normal serum lipid profiles. Furthermore, our results may explain why, with few exceptions (58), epidemiological studies have not shown a correlation between elevated plasma levels of total homocysteine and increased plasma levels of cholesterol and/or triglycerides.

Under normal circumstances, SREBP expression and activity are regulated by the SREBP cleavage-activation protein (SCAP) according to the lipid requirements of the cell $(59,60)$. Homocysteine appears to circumvent this feedback mechanism, maintaining cells in a sterolstarved state despite the accumulation of lipids. Although we do not currently understand how homocysteine dysregulates SREBP activation, the mechanism appears to involve homocysteine-induced ER stress. Recently it has been shown that ER stress induces cleavage of membrane-bound ATF6 by the same proteases that activate SREBP (designated S1P and S2P) (61). In addition, the ER stress-induced processing of ATF 6 does not require SCAP (61). These results raise the possibility that, in hepatocytes and smooth muscle cells, homocysteine-induced ER stress abrogates the requirement for SCAP and activates both ATF6 and SREBP by a similar mechanism. Identifying the components in the homocysteine-signaling cascade that ultimately regulate activation of SREBP and disrupt its feedback control system will further enhance our understanding of the link between hyperhomocysteinemia and the molecular events that result in increased lipid biosynthesis.

In conclusion, our data support a mechanism by which cells that are sensitive to elevated intracellular levels of homocysteine experience ER stress that leads to the activation and dysregulation of the endogenous sterol response pathway. In mice with diet-induced hyperhomocysteinemia this results in hepatic steatosis, a condition observed in virtually all CBS-deficient patients having severe hyperhomocysteinemia. Additional studies are now underway to determine whether this ER stress mechanism contributes to the development and progression of atherosclerotic lesions in hyperhomocysteinemia.

\section{Acknowledgments}

We thank Jack Hirsh and Jeffrey Weitz for helpful comments and suggestions throughout the course of these studies. This work was supported by Research Grant T4005 from the Heart and Stroke Foundation of Ontario (to R.C. Austin); NIH grant DK-58040 (to S.K. Krisans); NIH grants HL-63943, DK-25295, NS-24621, and an American Heart Association-Bugher Foundation Award (to S.R. Lentz); and the Office of Research and Development, Department of Veterans Affairs. Genotyping of CBS-deficient mice was performed by Lucy Robbins, Norma Sinclair, and Patricia Lovell in the University of Iowa Transgenic Facility under the direction of Curt D. Sigmund.

1. Ross, R. 1993. The pathogenesis of atherosclerosis: a perspective for the 1990s. Nature. 362:801-809.

2. McCully, K.S. 1996. Homocysteine and vascular disease. Nat. Med. 2:386-389.

3. Ueland, P.M., and Refsum, H. 1989. Plasma homocysteine, a risk factor for vascular disease: plasma levels in health, disease, and drug therapy. $J$. Lab. Clin. Med. 114:473-501.

4. Clarke, R., et al. 1991. Hyperhomocysteinemia: an independent risk factor for vascular disease. N. Engl. J. Med. 324:1149-1155.

5. Selhub, J., et al. 1995. Association between plasma homocysteine concentrations and extracranial carotid-artery stenosis. N. Engl. J. Med. 332:286-291.

6. Welch, G.N., and Loscalzo, J. 1998. Homocysteine and atherothrombosis. N. Engl. J. Med. 338:1042-1050.

7. den Heijer, M., et al. 1996. Hyperhomocysteinemia as a risk factor for deep vein thrombosis. N. Engl. J. Med. 334:759-762.

8. Wilken, D.E.L., and Dudman, N.P.B. 1992. Homocystinuria and atherosclerosis. In Molecular genetics of coronary artery disease. A.J. Lusis, J.I. Rotter, and R.S. Sparkes, editors. Karger. Basel, Switzerland. 311-324.

9. Harker, L.A., Slichter, S.J., Scott, C.R., and Ross, R. 1974. Homocystinemia: vascular injury and arterial thrombosis. N. Engl. J. Med. 291:537-543.

10. Mudd, S.H., Levy, H.L., and Skovby, F. 1989. Disorders of transsulfation. In The metabolic basis for inherited diseases. C.R. Scriver, A.L. Beadet, W.S. Sly, and D. Valle, editors. McGraw-Hill. New York, New York, USA. 693-734.

11. Gaull, G., Sturman, J.A., and Schaffner, F. 1974. Homocystinuria due to cystathionine synthase deficiency: enzymatic and ultrastructural studies. J. Pediatr. 84:381-390.

12. Watanabe, M., et al. 1995. Mice deficient in cystathionine $\beta$-synthase: animal models for mild and severe homocyst(e)inemia. Proc. Natl. Acad. Sci. USA. 92:1585-1589.

13. Harker, L.A., Harlan, J.M., and Ross, R. 1983. Effect of sulfinpyrazone on 
homocysteine-induced endothelial injury and arteriosclerosis in baboons. Circ. Res. 53:731-739.

14. Hladovec, J. 1979. Experimental homocysteinemia, endothelial lesions and thrombosis. Blood Vessels. 16:202-210.

15. Wall, R.T., Harlan, J.M., Harker, L.A., and Striker, G.E. 1980. Homocysteine-induced endothelial cell injury in vitro: a model for the study of vascular injury. Thromb. Res. 18:113-121.

16. Starkebaum, G., and Harlan, J.M. 1986. Endothelial cell injury due to copper-catalyzed hydrogen peroxide generation from homocysteine. $J$. Clin. Invest. 77:1370-1376.

17. deGroot, P.G., et al. 1983. Endothelial cell dysfunction in homocystinuria. Eur. J. Clin. Invest. 13:405-410.

18. Jakubowski, H., Zhang, L., Bardeguez, A., and Aviv, A. 2000. Homocysteine thiolactone and protein homocysteinylation in human endothelial cells. Implications for atherosclerosis. Circ. Res. 87:45-51.

19. Jakubowski, H. 1997. Metabolism of homocysteine thiolactone in human cells. Possible mechanism for pathological consequences of elevated homocysteine levels. J. Biol. Chem. 272:1935-1942.

20. Jacobsen, D.W. 2000. Hyperhomocysteinemia and oxidative stress. Time for a reality check? Arterioscler. Thromb. Vasc. Biol. 20:1182-1184.

21. Outinen, P.A., et al. 1999. Homocysteine-induced endoplasmic reticulum stress and growth arrest leads to specific changes in gene expression in human vascular endothelial cells. Blood. 94:959-967.

22. Outinen, P.A., et al. 1998. Characterization of the stress-inducing effects of homocysteine. Biochem. J. 332:213-221.

23. Kokame, K., Kato, H., and Miyata, T. 1996. Homocysteine-respondent genes in vascular endothelial cells identified by differential display analysis: GRP78 and novel genes. J. Biol. Chem. 271:29659-29665.

24. Kokame, K., Agarwala, K.L., Kato, H., and Miyata, T. 2000. Herp, a new ubiquitin-like membrane protein induced by endoplasmic reticulum stress. J. Biol. Chem. 275:32846-32853.

25. Cox, J.S., Chapman, R.E., and Walter, P. 1997. The unfolded protein response coordinates the production of endoplasmic reticulum protein and endoplasmic reticulum membrane. Mol. Biol. Cell. 8:1805-1814.

26. Travers, K.J., et al. 2000. Functional and genomic analyses reveal an essential coordination between the unfolded protein response and ERassociated degradation. Cell. 101:249-258.

27. Doerrler, W.T., and Lehrman, M.A. 1999. Regulation of the dolichol pathway in human fibroblasts by the endoplasmic reticulum unfolded protein response. Proc. Natl. Acad. Sci. USA. 96:13050-13055.

28. Brown, M.S., and Goldstein, J.L. 1999. A proteolytic pathway that controls the cholesterol content of membranes, cells, and blood. Proc. Natl. Acad. Sci. USA. 96:11041-11048

29. Horton, J.D., and Shimomura, I. 1999. Sterol regulatory element-binding proteins: activators of cholesterol and fatty acid synthesis. Curr. Opin. Lipidol. 10:143-150.

30. Jaffe, E.A., Nachmann, R.L., Becker, C.G., and Minnick, C.R. 1973. Culture of human endothelial cells derived from umbilical veins: identification by morphologic and immunologic criteria. J. Clin. Invest. 52:2745-2754.

31. Mudd, S.H., et al. 2000. Homocysteine and its disulfide derivatives: a suggested consensus terminology. Arterioscler. Thromb. Vasc. Biol. 20:1704-1706.

32. Lentz, S.R., et al. 2000. Folate dependence of hyperhomocysteinemia and vascular dysfunction in cystathionine $\beta$-synthase deficient mice. Am.J. Physiol. Heart Circ. Physiol. 279:H970-H975.

33. Malinow, M.R., et al. 1990. Homocyst(e)inemia in daily practice: levels in coronary artery disease. Coron. Artery Dis. 1:215-220.

34. Kuipers, F., et al. 1997. Impaired secretion of very low density lipoprotein-triglycerides by apolipoprotein E-deficient mouse hepatocytes. $J$. Clin. Invest. 100:2915-2922.

35. Lentz, S.R., et al. 1997. Consequences of hyperhomocyst(e)inemia on vascular function in atherosclerotic monkeys. Arterioscler. Thromb. Vasc. Biol. 17:2930-2934.

36. O, K., et al. 1998. Homocysteine stimulates the production and secretion of cholesterol in hepatic cells. Biochim. Biophys. Acta. 1393:317-324.

37. Brown, M.S., Faust, J.R., Goldstein, J.L., Kaneko, I., and Endo, A. 1978 Induction of 3-hydroxy-3-methylglutaryl coenzyme A reductase activity in human fibroblasts incubated with compactin (ML-236B), a compet- itive inhibitor of reductase. J. Biol. Chem. 253:1121-1128.

38. Amemiya-Kudo, M., et al. 2000. Promoter analysis of the mouse sterol regulatory element-binding protein (SREBP)-1c gene. J. Biol. Chem. 275:31078-31085.

39. Liu, H., Miller, E., van de Water, B., and Stevens, J.L. 1998. Endoplasmic reticulum stress proteins block oxidant-induced $\mathrm{Ca}^{2+}$ increases and cell death. J. Biol. Chem. 273:12858-12862.

40. Morris, J.A., Dorner, A.J., Edwards, C.A., Hendershot, L.M., and Kaufman, R.J. 1997. Immunoglobulin binding protein (BiP) function is required to protect cells from endoplasmic reticulum stress but is not required for the secretion of selective proteins. J. Biol. Chem. 272:4327-4334.

41. Wang, X.Z., et al. 1996. Signals from the stressed endoplasmic reticulum induce C/EBP-homologous protein (CHOP/GADD153). Mol. Cell. Biol. 16:4273-4280.

42. Wang, X.Z., et al. 1998. Cloning of mammalian Ire 1 reveals diversity in the ER stress responses. EMBO J. 17:5708-5717.

43. Selhub, J., Jacques, P.F., Wilson, P.W.F., Rush, D., and Rosenberg, I.H. 1993. Vitamin status and intake as primary determinants of homocysteinemia in an elderly population. JAMA. 270:2693-2698.

44. Robinson, K., et al. 1995. Hyperhomocysteinemia and low pyridoxal phosphate: common and independent reversible risk factors for coronary artery disease. Circulation. 92:2825-2830.

45. Ubbink, J.B., et al. 1996. The effect of a subnormal vitamin B-6 status on homocysteine metabolism. J. Clin. Invest. 98:177-184.

46. Rolland, P.H., et al. 1995. Hyperhomocysteinemia-induced vascular damage in the minipig. Circulation. 91:1161-1174.

47. Lentz, S.R., et al. 1996. Vascular dysfunction in monkeys with dietinduced hyperhomocyst(e)inemia. J. Clin. Invest. 98:24-29.

48. Loscalzo, J. 1996. The oxidant stress of hyperhomocyst(e)inemia. J. Clin. Invest. 98:5-7.

49. Eberhardt, R.T., et al. 2000. Endothelial dysfunction in a murine model of mild hyperhomocyst(e)inemia. J. Clin. Invest. 106:483-491.

50. Upchurch, G.R., et al. 1997. Homocyst(e)ine decreases bioavailable nitric oxide by a mechanism involving glutathione peroxidase. J. Biol. Chem. 272:17012-17017.

51. Lang, D., et al. 2000. Homocysteine-induced inhibition of endotheliumdependent relaxation in rabbit aorta: role for superoxide anions. Arterioscler. Thromb. Vasc. Biol. 20:422-427.

52. Li, L.J., et al. 1992. Establishment of a Chinese hamster ovary cell line that expresses grp78 antisense transcripts and suppresses A23187 induction of both GRP78 and GRP94. J. Cell. Physiol. 153:575-582.

53. Chapman, R., Sidrauski, C., and Walter, P. 1998. Intracellular signalling from the endoplasmic reticulum to the nucleus. Annu. Rev. Cell Dev. Biol. 14:459-485.

54. Pahl, H.L. 1999. Signal transduction from the endoplasmic reticulum to the cell nucleus. Physiol. Rev. 79:683-701.

55. Haze, K., Yoshida, H., Yanagi, H., Yura, T., and Mori, K. 1999. Mammalian transcription factor ATF6 is synthesized as a transmembrane protein and is activated by proteolysis in response to endoplasmic reticulum stress. Mol. Biol. Cell. 10:3787-3799.

56. Shimano, H., et al. 1996. Overproduction of cholesterol and fatty acids causes massive liver enlargement in transgenic mice expressing truncated SREBP-1a. J. Clin. Invest. 98:1575-1584.

57. Horton, J.D., Shimano, H., Hamilton, R.L., Brown, M.S., and Goldstein, J.L. 1999. Disruption of LDL receptor gene in transgenic SREBP-1a mice unmasks hyperlipidemia resulting from production of lipid-rich VLDL. J. Clin. Invest. 103:1067-1076.

58. Olszewski, A.J., and McCully, K.S. 1991. Homocysteine content of lipoproteins in hypercholesterolemia. Atherosclerosis. 88:61-68.

59. Nohturfft, A., Yabe, D., Goldstein, J.L., Brown, M.S., and Espenshade, P.J. 2000. Regulated step in cholesterol feedback localized to budding SCAP from ER membranes. Cell. 102:315-323.

60. Sakai, J., et al. 1996. Sterol-regulated release of SREBP-2 from cell membranes requires two sequential cleavages, one within a transmembrane segment. Cell. 85:1037-1046.

61. Ye, J., et al. 2000. ER stress induces cleavage of membrane-bound ATF6 by the same proteases that process SREBPs. Mol. Cell. 6:1355-1364. 